Review began 11/29/2021 Review ended 12/05/2021 Published 12/06/2021

\section{() Copyright 2021}

He et al. This is an open access article distributed under the terms of the Creative Commons Attribution License CC-BY 4.0. which permits unrestricted use, distribution, and reproduction in any medium, provided the original author and source are credited.

\title{
Bicuspid Aortic Valve in Pregnancy Complicated by Aortic Valve Vegetation, Aortic Root Abscess, and Aortic Insufficiency
}

\author{
Shan $\mathrm{He}^{1}$, Christopher A. Huynh ${ }^{1}$, Yi Deng ${ }^{1}$, Sandeep Markan ${ }^{1}$, Anvinh Nguyen ${ }^{1}$ \\ 1. Anesthesiology, Baylor College of Medicine, Houston, USA
}

Corresponding author: Shan He, shan.he@bcm.edu

\begin{abstract}
A 39-year-old patient presented to our Labor and Delivery unit with fever and nausea in the context of recent bacteriuria. She was found to be in sepsis due to an incidental bicuspid aortic valve (BAV) complicated by aortic valvular vegetations, severe aortic insufficiency, and aortic root abscess, requiring an emergent cesarean section. Three days after delivery, the patient successfully underwent a mechanical aortic valve replacement and root reconstruction. In this case report, medical, surgical, and anesthetic management of parturient patients with BAV are discussed. The management of this congenital valvulopathy and vasculopathy is complicated by the extensive hemodynamic and cardiovascular derangements that occur during pregnancy.
\end{abstract}

Categories: Anesthesiology, Cardiac/Thoracic/Vascular Surgery, Obstetrics/Gynecology

Keywords: infective endocarditis, aortic aneurysm, aortic valve insufficiency, congenital heart defects, bicuspid aortic valve disease

\section{Introduction}

Congenital bicuspid aortic valve (BAV) is the most common congenital heart malformation $[1,2]$. It is frequently associated with valvular complications, including aortic regurgitation, aortic stenosis (AS), and infective endocarditis, and vascular complications, including aortic dilatation, dissection, and aneurysm [25]. As more patients with congenital heart disease thrive in adulthood and consider pregnancy, anesthesia providers must be familiar with the pathophysiology and management of BAV. This article was previously presented as a poster at the 2021 American Society of Anesthesiologists (ASA) Anesthesiology Annual Meeting on October 10, 2021.

\section{Case Presentation}

A 39-year-old G5P2022 female at 34 weeks of pregnancy presented to the hospital for evaluation of a oneweek history of fever, chest pain, nausea, and vomiting. Her pregnancy had been complicated by two prior hospital visits for similar symptoms for Group B streptococcal (GBS) bacteriuria. The patient did not have a history of intravenous drug abuse.

On admission to the Labor and Delivery Unit, her vitals were notable for maternal tachycardia up to 130 beats per minute (BPM), fetal tachycardia up to 200 BPM, maternal hypotension with blood pressure (BP) of 84/49 $\mathrm{mmHg}$, and intermittent desaturations to $94 \%$ on room air. She continued to endorse subjective fever, malaise, and dyspnea. Her initial laboratory workup revealed leukocytosis of $16.7 \times 10^{9} / \mathrm{L}$ and thrombocytopenia of $68 \times 10^{9} / \mathrm{L}$. Blood cultures eventually revealed Streptococcus agalactiae (GBS) bacteremia, suspected due to a urinary source.

A transthoracic echocardiogram (TTE) was performed to evaluate for endocarditis. TTE demonstrated a $1.2 \times$ $1.0 \mathrm{~cm}$ vegetation on the aortic valve, specifically on the ventricular aspect of the anatomical left coronary cusp. This cusp was noted to prolapse into the left ventricular outflow tract (LVOT) causing severe eccentric severe aortic insufficiency (AI). In addition, there was mild-to-moderate AS with a peak velocity of $3.1 \mathrm{~m} / \mathrm{s}$, a mean gradient of $18 \mathrm{mmHg}$, and an area of $1.0 \mathrm{~cm}^{2}$.

A multidisciplinary team including cardiothoracic surgery, cardiac anesthesiology, and maternal-fetal medicine was convened. The team elected to proceed with emergent cesarean delivery (CS) under general anesthesia to facilitate intraoperative transesophageal echocardiogram (TEE).

A preoperative radial arterial line was placed for closer hemodynamic monitoring. Induction was achieved with $0.2 \mathrm{mg} / \mathrm{kg}$ of etomidate, $1.3 \mathrm{mg} / \mathrm{kg}$ of lidocaine, $1.7 \mathrm{mg} / \mathrm{kg}$ of propofol, and $1.5 \mathrm{mg} / \mathrm{kg}$ of succinylcholine, followed by easy intubation. The patient remained hemodynamically stable throughout the case, and the delivery was uneventful. Intraoperative TEE demonstrated a bicuspid aortic valve with diffuse, bulky vegetations studding both leaflets protruding into the LVOT, with the largest vegetation measuring 2 


\section{Cureus}

$\mathrm{cm}$ in length (Figures 1,2). The leaflets were perforated at two locations, leading to mild AS and severe AI; vena contracta of the two regurgitant jets measured $0.8 \mathrm{~cm}$ and $0.4 \mathrm{~cm}$. A small aortic root abscess was also discovered on the mitral side of the aortic valve. The mitral valve did not have vegetations. The patient was extubated without complications.

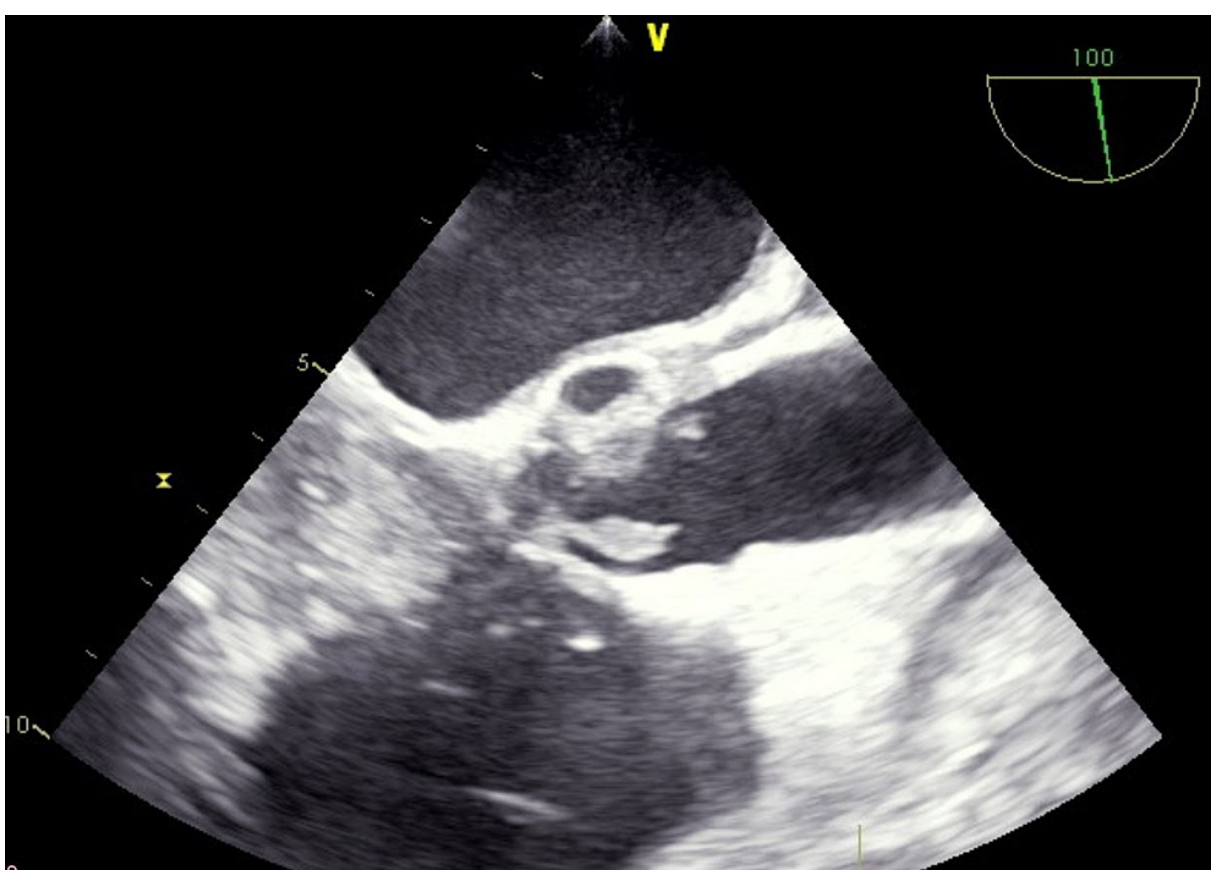

FIGURE 1: Transesophageal image at the mid-esophageal aortic valve long-axis view demonstrating diffuse bulky vegetations.

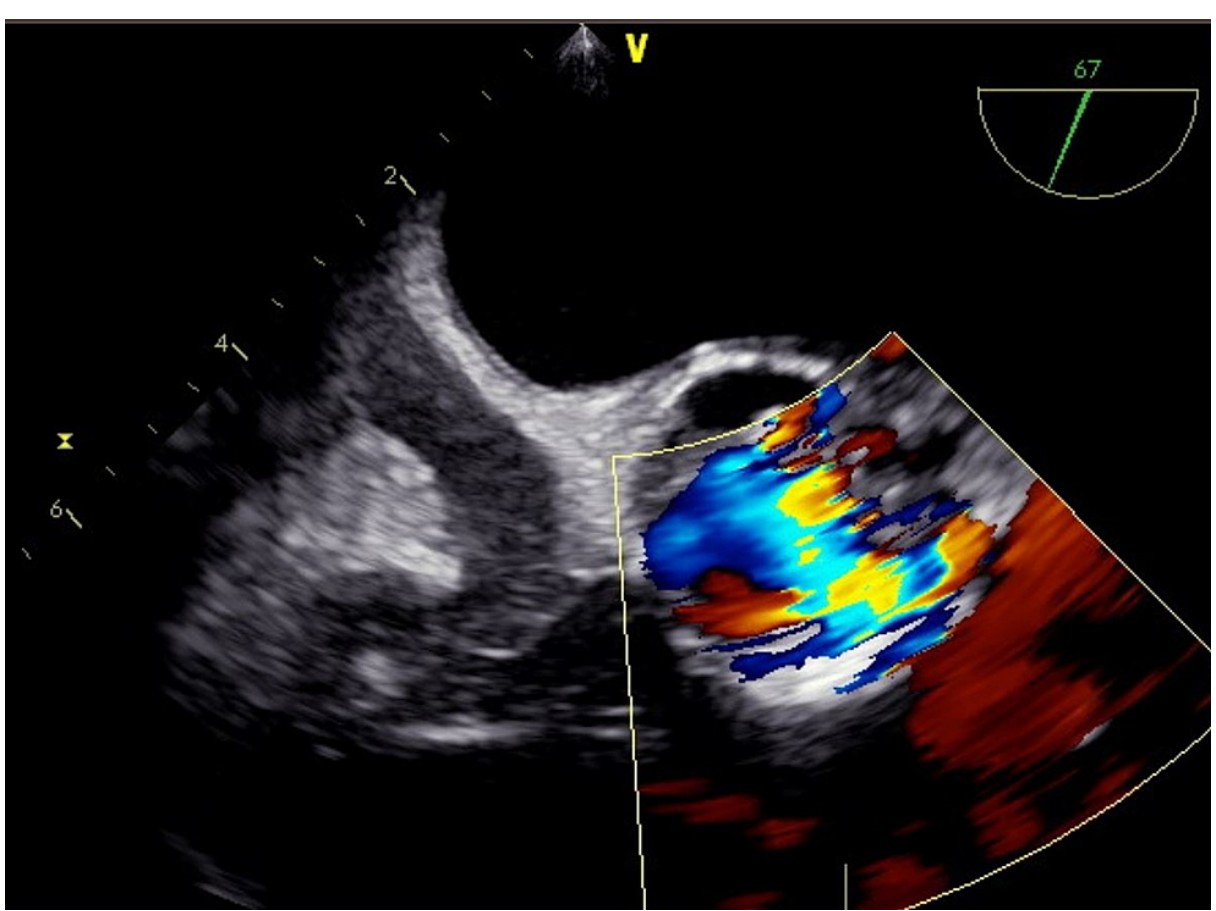

FIGURE 2: Transesophageal images at the mid-esophageal aortic valve short-axis view with the color Doppler box positioned over the aortic valve demonstrating continuous flow during systole and diastole, indicative of aortic insufficiency. 
Two days after delivery, the patient decided to undergo mechanical aortic valve replacement (17-mm St. Jude Medical Hemodynamic Plus valve), aortic annular enlargement, subannular abscess debridement, and aortic root reconstruction with bovine pericardium. Surgical findings included pericardial purulence and large-seeded vegetation on the bicuspid aortic valve. The root abscess tracked into the fibrosa of the heart, posterior aorta-ventricular wall, and the dome of the left atrium requiring extensive debridement. The patient's chest was left open and packed given concerns of continued bleeding after extensive debridement. She was closed on postoperative day six after multiple washouts. The patient was extubated on postoperative day nine and discharged on postoperative day 25 .

\section{Discussion}

\section{The condition}

Congenital BAV has an incidence of $1-2 \%$ in the general population and is the most common congenital valve malformation $[1-3,5,6]$. The exact pathogenesis is unknown but is theorized to be due to aberrant aortic cusp development during valvulogenesis. This leads to a "bicuspid" conformation of unequal cusp sizes when two cusps fuse to form one larger leaflet [5-7]. In addition, BAV is frequently associated with aortic pathologies, including root and ascending aortic dilatation, interrupted and hypoplastic arch, coarctation of the aorta, patent ductus arteriosus, and coronary vasculature abnormalities, suggesting shared developmental pathogenesis of the aorta and aortic valve $[2,5-8]$.

In patients afflicted with $\mathrm{BAV}$, there is likely a genetic defect in collagen and elastin metabolism with progressive valvular and vascular complications. The connective tissue properties in these patients are inherently abnormal due to upregulated matrix metalloproteinases and apoptotic smooth muscle cells, drawing clinical parallels to other connective tissue diseases such as Marfan's and Ehlers-Danlos disease $[5,7]$. It is this heritable primary aortopathy with chronic inflammation, deterioration, and abnormal remodeling of the aortic media that lend to an increased propensity for aortic dilatation, dissection, and aneurysm in $30-50 \%$ of BAV patients $[4,5,7,8]$.

Valvular complications associated with BAV include AS, AI, and infective endocarditis. Stenosis occurs from premature fibrosis and accelerated calcification of the abnormal leaflets, regurgitation from prolapse, fibrotic retraction of the aberrant cusps, and annular dilation [5-8]. Valvular complications often worsen in pregnancy, leading to heart failure, arrhythmias, and myocardial infarction [4].

Infective endocarditis typically involves anatomically abnormal cardiac valves and occurs in 1:8,000 of all pregnancies [9-11]. In BAV, susceptible endothelium allows platelets and fibrin thrombi to form, creating a nidus for seeding and proliferation of microbial organisms [9]. Vegetations not only lead to AS/AI their dislodgement can cause acute vascular insufficiencies such as myocardial, pulmonary infarctions, limb ischemia, or strokes $[11,12]$. Surgery is indicated if severe and progressive symptoms, heart failure, embolic events, or abscess leading to valve dehiscence are present [12].

\section{Physiologic changes of pregnancy and its impact on bicuspid aortic valve management}

Pregnancy causes profound physiologic changes that compound pre-existing hemodynamic risk factors in patients with BAV. Cardiac output (CO) progressively increases throughout pregnancy and peaks at the 36th week of gestation, reaching $130-150 \%$ of pre-pregnancy capacity $[2,10,13]$. This hyperdynamic state subsequently leads to increased vascular wall tension, imposing additional shearing force on the aorta $[1,13]$.

In addition, hormonal changes also cause weakening in the aortic wall. Estrogen and progesterone induce histological changes similar to that cystic medial necrosis in the tunica media and intima $[1,4,14]$. Thus, pregnancy has historically been associated with an increased risk of aortic dissection, which is especially pronounced in those with an aortopathy $[1,3]$.

\section{Medical and surgical management of bicuspid aortic valve}

Parturient patients with BAV can experience safe pregnancies [3]. The key factor for risk stratification is the aortic diameter, with $\leqslant 4.5 \mathrm{~cm}$ indicating lower risk. Stringent hemodynamic regulation with beta-blockade and serial echographic monitoring is advised to rule out further cardiac dysfunction and progression in aortic diameter $[3,4,14]$.

Approximately one-third of BAV patients ultimately undergo surgery involving the valve, aorta, or both [3]. AS or AI leading to critical narrowing, heart failure, or severe clinical symptoms are common surgical indications $[2,15]$. Concomitant replacement of the ascending aorta should be performed if the diameter exceeds $4.5 \mathrm{~cm}[2,16]$. The aortic root can be spared if no dilatation is present, but prophylactic surgery before pregnancy should be considered if the diameter exceeds $\geqslant 5.0 \mathrm{~cm}[2,13,14]$.

\section{Anesthetic management of bicuspid aortic valve during pregnancy}


Pregnancy in BAV patients is typically well-tolerated barring severe AS or AI leading to heart failure or significant aortic dilation exceeding $\geqslant 4.5 \mathrm{~cm}$. Similar to patients without BAV, controlled vaginal delivery with epidural anesthesia and instrument assistance during the second stage is the preferred mode of delivery due to decreased risk of hemodynamic shifts with vaginal deliveries $[2,4,14]$. Neuraxial anesthesia can also modulate the maternal pain response during parturition; the sympathectomy can lead to decreased systemic vascular resistance and decreased venous return and preload. Its effects can assist in minimizing aortic wall stress and the effect of CO augmentation during contractions [4]. General anesthesia and CS are considered in severe valvulopathy, aortic disease with a high risk of rupture, or dissection to avoid increased CO in the context of uterine contractions [4]. BP and heart rate (HR) must be carefully monitored, especially during the peripartum period, given that the risk of aortic dissection is the highest late during gestation and shortly after delivery [2].

Given our patient's profound thrombocytopenia, the risk of epidural hematoma outweighed the benefits of the neuraxial technique. We elected to perform general anesthesia for the CS to facilitate the use of intraoperative TEE. HR was maintained above 80 BPM to reduce the regurgitant fraction and BP was carefully titrated to reduce shear stress on the aorta while ensuring adequate uterine blood flow for fetal perfusion. Although preload is critical in patients with AS, we chose to be judicious with our fluid resuscitation, given the hypervolemic state associated with pregnancy. As the patient remained hemodynamically stable throughout the CS and TEE did not indicate worsening heart failure, we elected to extubate the patient with admission to the intermediate care unit before her cardiac surgery two days later.

\section{Conclusions}

BAV is a complex vascular disease with an abnormality of connective tissue, specifically implicating the aortic valve and the ascending aorta. BAV is also rarely a benign disease because given time every individual with a BAV will develop AS and/or AI due to the anatomical deviation of the leaflets. It often causes significant morbidity and mortality in the peripartum population. Physiologic blood volume, HR, stroke volume, and aortic root size are all increased in pregnancy which further exacerbate the underlying condition. Clinical management is fundamentally challenging and must be individualized. Given thoughtful medical, surgical, and anesthetic management of pregnant patients with BAV, they can often experience safe pregnancies.

\section{Additional Information \\ Disclosures}

Human subjects: Consent was obtained or waived by all participants in this study. Conflicts of interest: In compliance with the ICMJE uniform disclosure form, all authors declare the following: Payment/services info: All authors have declared that no financial support was received from any organization for the submitted work. Financial relationships: All authors have declared that they have no financial relationships at present or within the previous three years with any organizations that might have an interest in the submitted work. Other relationships: All authors have declared that there are no other relationships or activities that could appear to have influenced the submitted work.

\section{Acknowledgements}

Drs He, Huynh, Deng, Markan, and Nguyen would like to thank the Anesthesiology Department at Baylor College of Medicine for their continued academic support.

\section{References}

1. Anderson RA, Fineron PW: Aortic dissection in pregnancy: importance of pregnancy-induced changes in the vessel wall and bicuspid aortic valve in pathogenesis. Br J Obstet Gynaecol. 1994, 101:1085-8. 10.1111/j.1471-0528.1994.tb13589.x

2. Friedman T, Mani A, Elefteriades JA: Bicuspid aortic valve: clinical approach and scientific review of a common clinical entity. Expert Rev Cardiovasc Ther. 2008, 6:235-48. 10.1586/14779072.6.2.235

3. McKellar SH, MacDonald RJ, Michelena HI, Connolly HM, Sundt TM 3rd: Frequency of cardiovascular events in women with a congenitally bicuspid aortic valve in a single community and effect of pregnancy on events. Am J Cardiol. 2011, 107:96-9. 10.1016/j.amjcard.2010.08.061

4. Smok DA: Aortopathy in pregnancy. Semin Perinatol. 2014, 38:295-303. 10.1053/j.semperi.2014.04.019

5. Fedak PW, Verma S, David TE, Leask RL, Weisel RD, Butany J: Clinical and pathophysiological implications of a bicuspid aortic valve. Circulation. 2002, 106:900-4. 10.1161/01.cir.0000027905.26586.e8

6. Ward C: Clinical significance of the bicuspid aortic valve. Heart. 2000, 83:81-5. 10.1136/heart.83.1.81

7. Braverman AC, Güven H, Beardslee MA, Makan M, Kates AM, Moon MR: The bicuspid aortic valve. Curr Probl Cardiol. 2005, 30:470-522. 10.1016/j.cpcardiol.2005.06.002

8. Keane MG, Wiegers SE, Plappert T, Pochettino A, Bavaria JE, Sutton MG: Bicuspid aortic valves are associated with aortic dilatation out of proportion to coexistent valvular lesions. Circulation. 2000, 102:III35-9. 10.1161/01.cir.102.suppl_3.iii-35

9. Campuzano K, Roqué H, Bolnick A, Leo MV, Campbell WA: Bacterial endocarditis complicating pregnancy: case report and systematic review of the literature. Arch Gynecol Obstet. 2003, 268:251-5. 10.1007/s00404003-0485-x 


\section{Cureus}

10. Nazarian M, McCullough GH, Fielder DL: Bacterial endocarditis in pregnancy: successful surgical correction . J Thorac Cardiovasc Surg. 1976, 71:880-3. 10.1016/S0022-5223(19)40125-6

11. Cox SM, Hankins GD, Leveno KJ, Cunningham FG: Bacterial endocarditis. A serious pregnancy complication . J Reprod Med. 1988, 33:671-4.

12. Kebed KY, Bishu K, Al Adham RI, et al.: Pregnancy and postpartum infective endocarditis: a systematic review. Mayo Clin Proc. 2014, 89:1143-52. 10.1016/j.mayocp.2014.04.024

13. Lansman SL, Goldberg JB, Kai M, Tang GH, Malekan R, Spielvogel D: Aortic surgery in pregnancy. J Thorac Cardiovasc Surg. 2017, 153:S44-8. 10.1016/j.jtcvs.2016.06.015

14. Wanga S, Silversides C, Dore A, de Waard V, Mulder B: Pregnancy and thoracic aortic disease: managing the risks. Can J Cardiol. 2016, 32:78-85. 10.1016/i.cjca.2015.09.003

15. Bonow RO, Carabello BA, Kanu C, et al.: ACC/AHA 2006 guidelines for the management of patients with valvular heart disease: a report of the American College of Cardiology/American Heart Association Task Force on Practice Guidelines (writing committee to revise the 1998 Guidelines for the Management of Patients With Valvular Heart Disease): developed in collaboration with the Society of Cardiovascular Anesthesiologists: endorsed by the Society for Cardiovascular Angiography and Interventions and the Society of Thoracic Surgeons. Circulation. 2006, 114:e84-231. 10.1161/CIRCULATIONAHA.106.176857

16. Borger MA, Fedak PW, Stephens EH, et al.: The American Association for Thoracic Surgery consensus guidelines on bicuspid aortic valve-related aortopathy: executive summary. J Thorac Cardiovasc Surg. 2018, 156:473-80. 10.1016/i.jtcvs.2017.10.161 